\title{
Flash Evaporation as an Attempt for Preparation of YBCO Superconductor Thin Films
}

\author{
A. A. Ramadan, S. Abdel-Hady, S. Abdel-Ghany and S. E. Soltan \\ Physics Department, Faculty of Science, Helwan University, Helwan, Cairo, \\ Egypt.
}

A home-made flash evaporation attachment was used to prepare YBCO superconductor in the form of thin film on Si wafer substrate. Structural study was achieved by XRD analysis and electric resistance was measured by linear four-probe method down to $77 \mathrm{~K}$. The whole-pattern fitting of Rietveld method was applied to verify the presence of the orthorhombic YBCO phase in the prepared bulk sample used as a source for thin film deposition. Thin films deposited onto Si(111) wafer showed crystalline phase in case of substrate temperature $\geq 450{ }^{\circ} \mathrm{C}$ and post annealing at temperature $\geq 850{ }^{\circ} \mathrm{C}$. The best and unexpected characteristics $\left(T_{c}=125 \mathrm{~K}, \Delta T=0.5 \mathrm{~K}\right)$ were obtained at critical thickness of $85 \mathrm{~nm}$ for films deposited at substrate temperature of 500 ${ }^{\circ} \mathrm{C}$ and subjected to post annealing in oxygen at $950{ }^{\circ} \mathrm{C}$. This was attributed to the preferred orientation (c-axis normal to the substrate) and to the optimum Si concentration by interdiffusion under the proposed preparation condition. Increasing the film thickness than $100 \mathrm{~nm}$ deteriorates the superconducting behaviour. On the other hand, films of thickness less than $80 \mathrm{~nm}$ did not show superconducting behaviour. 


\section{Introduction}

Many applications of high temperature superconductors (HTSC) [1] in advanced technology usually require the superconductor in the form of thin films. There has been rapid progress in the fabrication of HTSC thin films on oriented substrate materials such as $\mathrm{SrTiO}, \mathrm{MgO}$ and $\mathrm{LaAlO}_{3}$. Recently, more attention has been given to silicon substrate because of its advantages such as high thermal conductivity and compatibility to semiconductor devices [2]. However, the obtained results have been less impressive and the quality of the HTSC thin films has not been satisfactory [3] due to the strong interdiffusion between the superconducting material and the substrate. This problem was partially solved by employing appropriate single [4] or double [2,5] buffer layer systems but there remain some problems. Varieties of thin film preparation methods are widely used, nevertheless, the flash evaporation technique is not greatly considered in the literature as, for example, the sputtering one.

This work is an attempt to prepare YBCO superconductor in the form of thin films to using a home-made flash evaporation attachment. Bulk sample was prepared and characterized in order to be used as the source material. Structural study by X-ray diffraction (XRD) and the whole-pattern fitting of Rietveld method [9] will be applied. The electric resistance measurement will be used to determine the critical temperature.

\section{Experimentation and Characterization}

\section{Sample preparation:}

The source material for the deposition of thin films was prepared by the solid-state technique. Samples of $\mathrm{YBa}_{2} \mathrm{Cu}_{3} \mathrm{O}_{7}$ were prepared by the reaction between the raw materials $\mathrm{Y}_{2} \mathrm{O}_{3}, \mathrm{BaO}$ and $\mathrm{CuO}$ of high purity (99.99\%) in the desired atomic ratio (1:2:3). The corresponding weight ratio (1:3:2) was ground carefully to get proper mixing and to facilitate the calcination process. An appropriate preparation scheme of pressing into disc shape pellets, sintering, regrinding, pelletizing, sintering in air and oxygen was considered. The prepared samples were used for the subsequent preparation of thin films.

Although some limitation of the flash evaporation technique, it has attractive features [7]. By careful management, the difficulties can be overcome, and deposited films of splatter-free surface can be achieved. A sketch of the home-made flash evaporation attachment is depicted in Fig.(1). The evaporation (boat) temperature was kept constant at about $1350{ }^{\circ} \mathrm{C}$ and the deposition rate has been controlled at a mean value of $1 \mathrm{~nm} \mathrm{~s}^{-1}$ by the vibrator 
of the home-made flash evaporation attachment. Two processes were considered; ex-situ and in-situ preparation. The first step in ex-situ (or post annealing) process is the formation of an amorphous or polycrystalline film of the components with the appropriate stoichiometry. The next step is the annealing in flowing oxygen inside a tube-furnace to crystallize the film and/or adjust its oxygen content to optimize the superconducting properties. In case of in-situ process, film production involves deposition of the materials onto substrates at high temperature. The substrate temperatures were $28,400,450$ and $500{ }^{\circ} \mathrm{C}$. The deposition is followed by annealing at 750,850 and $950{ }^{\circ} \mathrm{C}$ for $5 \mathrm{hr}$, then cooling in oxygen atmosphere to form the fully oxygenated orthorhombic superconducting phase.

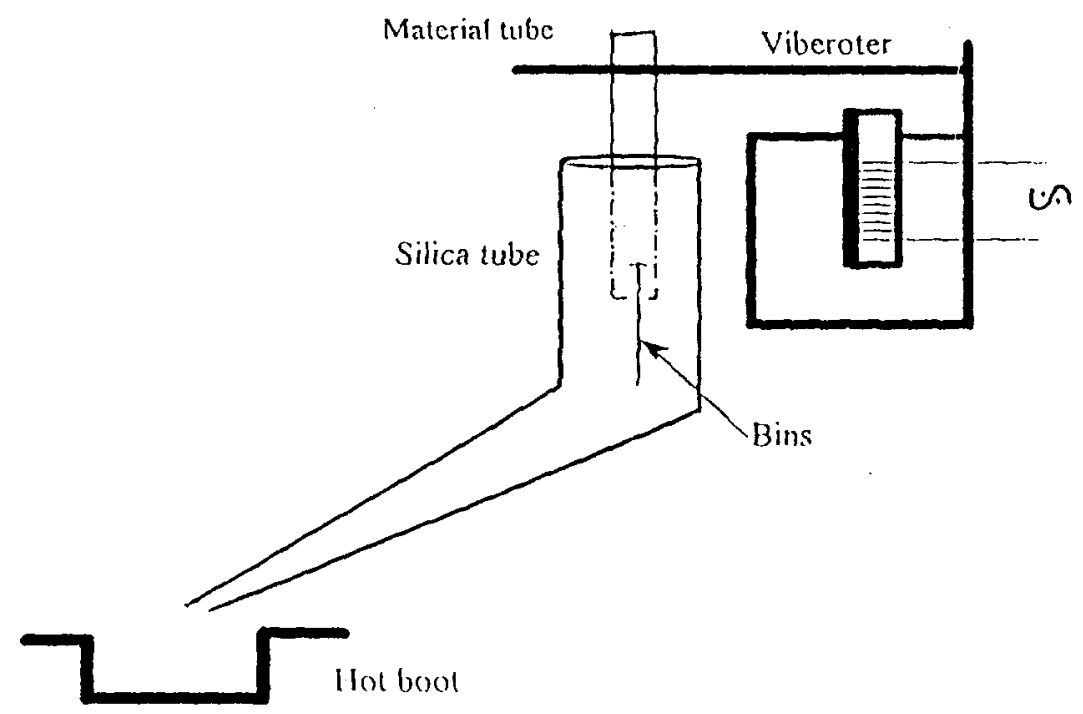

Fig. (1) : Sketch of the flash evaporation attachment.

\section{Sample Characterization:}

$X$-ray diffraction analysis:

The microstructure characteristics (material state, preferred orientation and phase identification) of the prepared sample of YBCO in the form of powder (bulk) or films were investigated by X-ray diffraction (XRD) analyses using reflecting geometry and $\mathrm{Cu}-\mathrm{K} \alpha$ radiation. The mode of continuos scan was used in the range of $2 \theta=20-60^{\circ}$ for phase identification and the stepscanning mode for refinement by Rietveld method. The step size was $0.05^{\circ}(2 \theta)$ and the counts were collected for $10 \mathrm{~s}$ at each step. 
Thickness measurement:

Film thickness was measured by quartz-crystal for monitoring and controlling of the deposition rate as well as the thickness during film preparation, and the multiple-beam Fizeau method at reflection after the preparation of the films. A set-up of the interferometer was constructed and used successfully down to about $8 \mathrm{~nm}$.

\section{Resistance measurements:}

A compact liquid nitrogen Oxford cryostat, type DN1704, was used for resistance measurements. The temperature is obtained and maintained constant automatically by the heater mounted on the heat exchanger. The resistivity measurements, either for the bulk or thin films, were done by the linear fourprobe method using spring loaded pines and silver paint for the electrical contacts. The voltage is measured by Keithley 617 electrometer and the current density during the measurement was $7.2+1.6 \mathrm{~A} / \mathrm{cm}^{2}$ The criteria used to define the transition temperature $T_{c}$ is the midpoint value determined by the point at which the first derivative of the resistivity curve reaches its maximum [8].

\section{Results and Discussion:}

\section{Source material:}

XRD investigation of the prepared bulk material was carried out in order to verify the presence of the orthorhombic YBCO phase. The wholepattern fitting of Rietveld method was applied to the obtained diffraction data using DBWS-9411 program written by Young et al. [14]. An acceptable fitting with $\mathrm{Rw} \%=9.2$ and goodness of fit $\mathrm{s}=1.83$ were achieved. The observed (dotted) and the calculated (continuos line) data as well as their difference (in the lower field) are depicted in Fig.(2). The presence of mainly the $\mathrm{YBa}_{2} \mathrm{Cu}_{3} \mathrm{O}_{7^{-}}$ ${ }_{\delta}$ orthorhombic phase was proved with unit cell parameters of $\mathrm{a}=0.383 \mathrm{~nm}$, $\mathrm{b}=0.388 \mathrm{~nm}$ and $\mathrm{c}=1.169 \mathrm{~nm}$.

The temperature dependence of the normalized resistance $(\mathrm{R}(\mathrm{T}) / \mathrm{R}(300 \mathrm{~K}))$ is shown as R-T curve in Fig.(3). The on-set temperature of the superconductivity transition was at $90 \mathrm{~K}$ and the zero point at $89 \mathrm{~K}$. The $\mathrm{T}_{\mathrm{c}}$ of $89.5 \mathrm{~K}$ was measured at the peak maximum of the first derivative of the R-T curve [8]. This is the normal expected value for YBCO material in the bulk form [10]. Thus, the sharp transition width and the value of $T_{c}$ verify the structural study indicating the presence of the orthorhombic YBCO phase. Therefore, the prepared sample can be used as a source material for thin film preparation. 


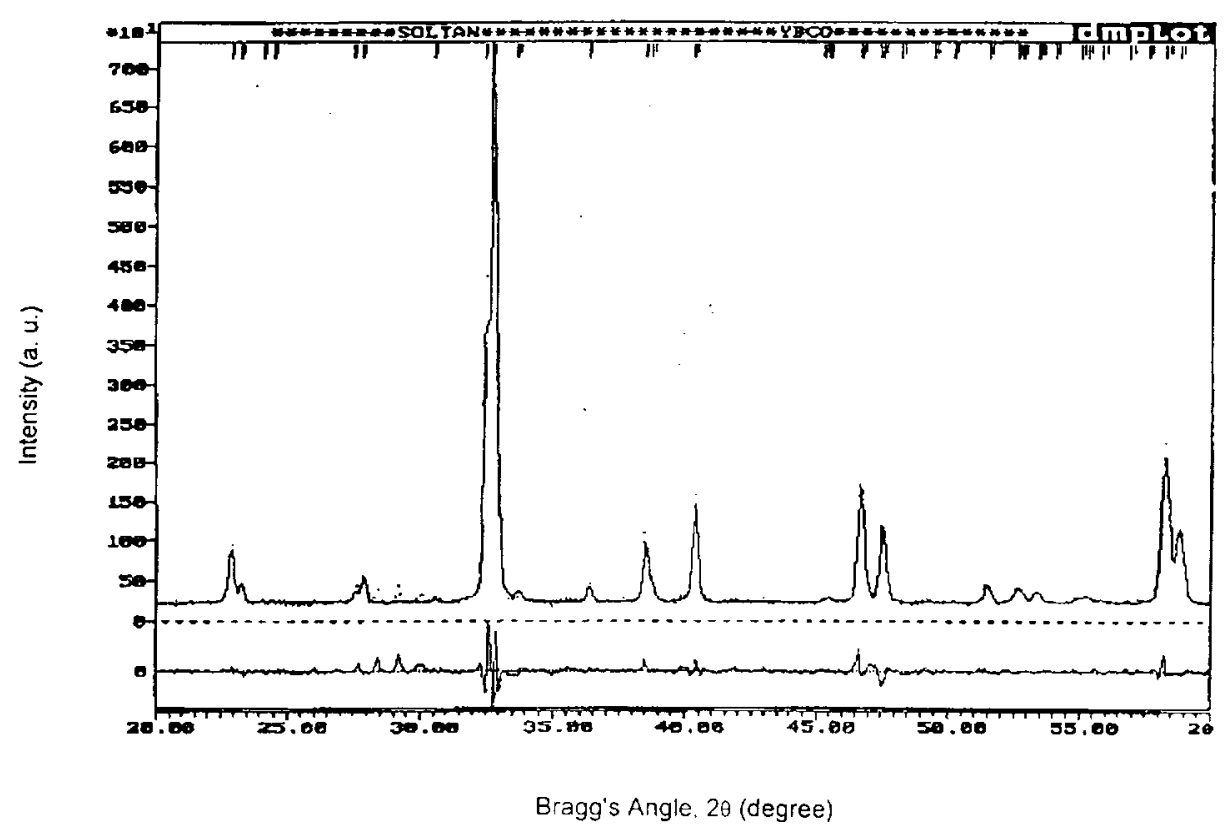

Fig. (2) : Rietveld refinement of the XRD patterns of YBCO bulk material.

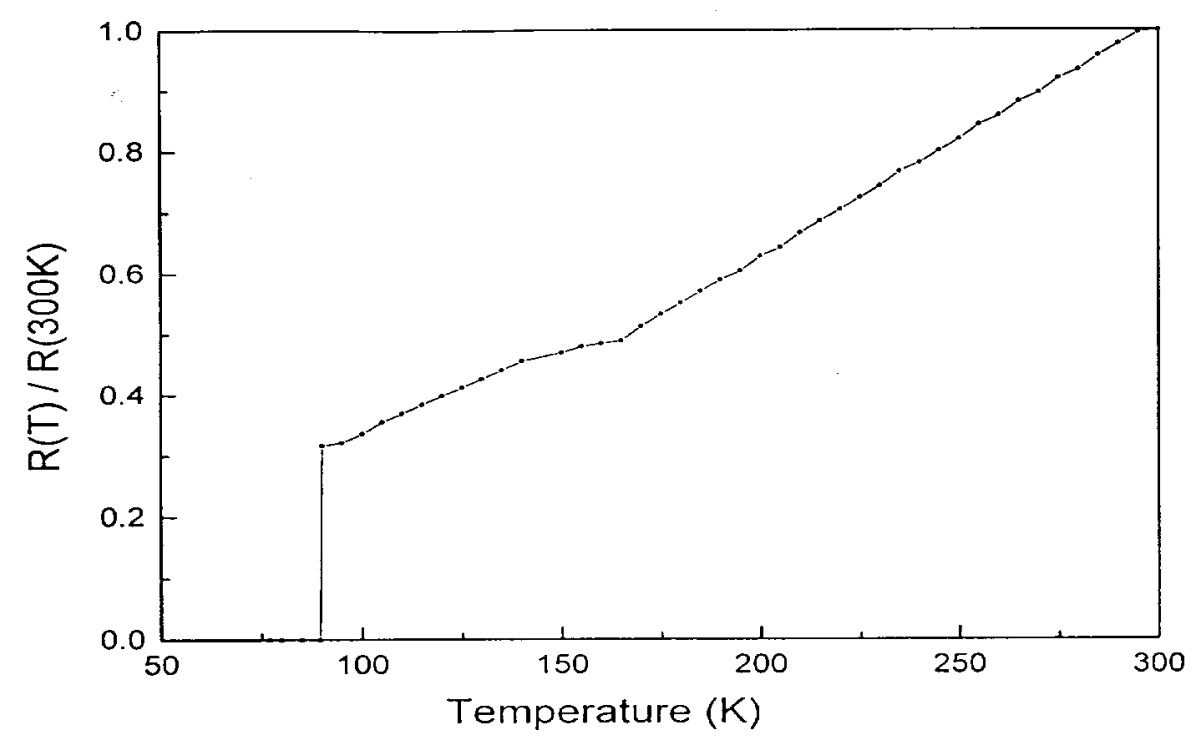

Fig. (3) : Temperature dependence of normalized resistance for the prepared YBCO bulk material 
Thin Films:

Using silicon wafer of (111) surface gives the possibility to deposit the films at higher substrate temperatures. Also, epitaxial growth can be achieved; such preferred orientation may enhance the superconducting characteristics of the deposited films. In this work, three different series of samples were deposited with the same preparation condition but at different substrate temperatures. Samples within each series were annealed at different temperatures in addition to the as deposited one.

Clearly, the obtained diffractograms of the prepared films include also the diffraction patterns of the substrate. The diffraction line of the maximum intensity of the silicon wafer is observed in a $2 \theta$ range, unfortunately, where the characteristic lines of (123) YBCO appear; this makes the identification a difficult task. Combination of substrate temperature, $\mathrm{T}_{\mathrm{s}}$, and annealing one, $\mathrm{T}_{\mathrm{a}}$, bring about three types of XRD patterns indicating the different structural characteristics of the deposited materials. These cases are summariezed in Table(1).

Table (1):

Conditions of heat treatment for different types of XRD patterns.

\begin{tabular}{|c|c|c|}
\hline \multirow{2}{*}{$\mathbf{T}_{\mathbf{s}}\left({ }^{\mathbf{}} \mathbf{C}\right)$} & $\mathbf{T}_{\mathbf{a}}\left({ }^{\mathbf{}} \mathbf{C}\right)$ & XRD \\
\hline \multirow{3}{*}{$\leq 200$} & No & N \\
\cline { 2 - 2 } & 750 & \multirow{2}{*}{ W } \\
& 850 & \\
\hline \multirow{3}{*}{$\geq 450$} & 950 & \\
& No & \\
\cline { 2 - 3 } & 750 & \multirow{2}{*}{$\mathrm{S}$} \\
\hline
\end{tabular}

$\mathbf{N}=$ No diffraction lines

$\mathrm{W}=$ Weak diffraction lines

$\mathrm{S}=$ Strong diffraction lines

The best results were for films deposited at $\mathrm{T}_{\mathrm{s}} \geq 450{ }^{\circ} \mathrm{C}$ and then subjected to post annealing at $\mathrm{T}_{\mathrm{a}} \geq 850{ }^{\circ} \mathrm{C}$ for $5 \mathrm{hr}$ : additional strong diffraction 
(S.L.) lines were observed as shown in Fig.(4) due to high degree of crystallization. The observed d-spacing (those of Si are not included) of films deposited under the above conditions are given in Table (2). Identification indicated that the presence of the orthorhombic (123) YBCO phase (ICDD-data file no. 39-486) is the most probable one; the corresponding hkl indices are given also in Table (2). The relative intensities of diffraction patterns were not easy to be estimated due to the overlap with those of $\mathrm{Si}$ wafer. However, it is worth to mention that the peak attributed to $006(\mathrm{~d}=0.1976 \mathrm{~nm})$ is the strongest one, which indicates that the prepared film is oriented with the c-axis normal to the substrate. This preferred orientation, clearly, enhance the superconducting behaviour. The average value of c-parameter was calculated as $1.198 \mathrm{~nm}$ from the $00 l$ diffraction lines [(003), (004) and (004)]. The observed increase in this value than that of the bulk can not be explained by the expecting silicon doping by interdiffusion but may be a result of the preferential growth of the film with the c-direction perpendicular to its surface and the compression stresses in $\mathrm{a}-\mathrm{b}$ plane.

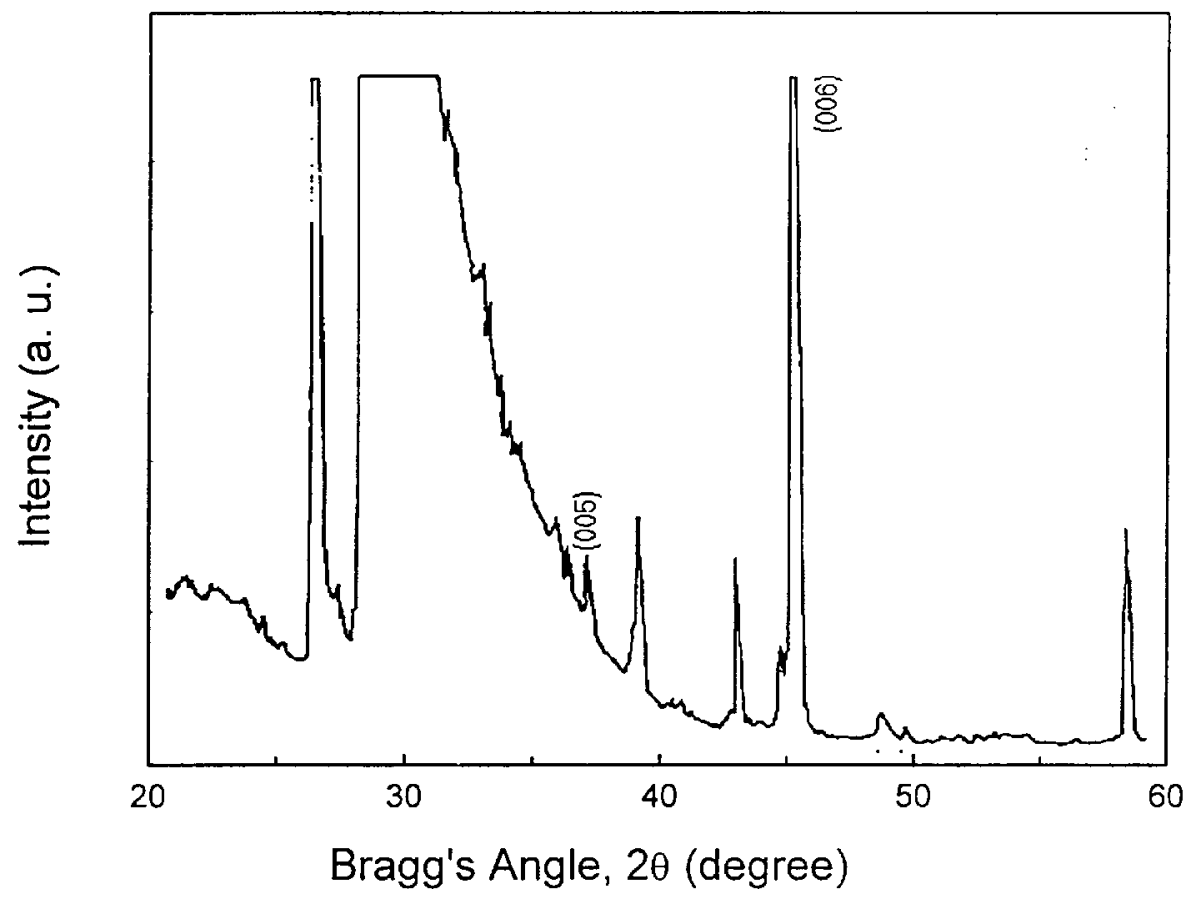

Fig. (4) : X-ray diffractograms of deposited film on Si single crystal substrate $\left(\mathrm{T}_{\mathrm{s}}=500{ }^{\circ} \mathrm{C}, \mathrm{T}_{\mathrm{a}}=850{ }^{\circ} \mathrm{C}\right)$. 
Table (2):

XRD data of films deposited on Si single crystal substrate at $T_{s}=500{ }^{\circ} \mathrm{C}$ and annealed at $\mathrm{T}_{\mathrm{a}}=850{ }^{\circ} \mathrm{C}$.

\begin{tabular}{|c|c|}
\hline $\mathbf{d}(\mathbf{n m})$ & hkl \\
\hline 0.3767 & 003 \\
\hline 0.2736 & 013 \\
\hline 0.2712 & 103,110 \\
\hline 0.2506 & 112 \\
\hline 0.2428 & 005 \\
\hline 0.2021 & 105 \\
\hline 0.1976 & 006 \\
\hline
\end{tabular}

Ascending the substrate temperature to $\mathrm{T}_{\mathrm{s}} \geq 450{ }^{\circ} \mathrm{C}$, annealing starts to affect the superconducting behaviour of the prepared films. Annealing at $\mathrm{T}_{\mathrm{a}} \leq$ $750{ }^{\circ} \mathrm{C}$ showed metallic behaviour without any superconducting transition down to $\mathrm{T} \geq 77 \mathrm{~K}$ as shown in Fig.(5). This is in accordance with the structural

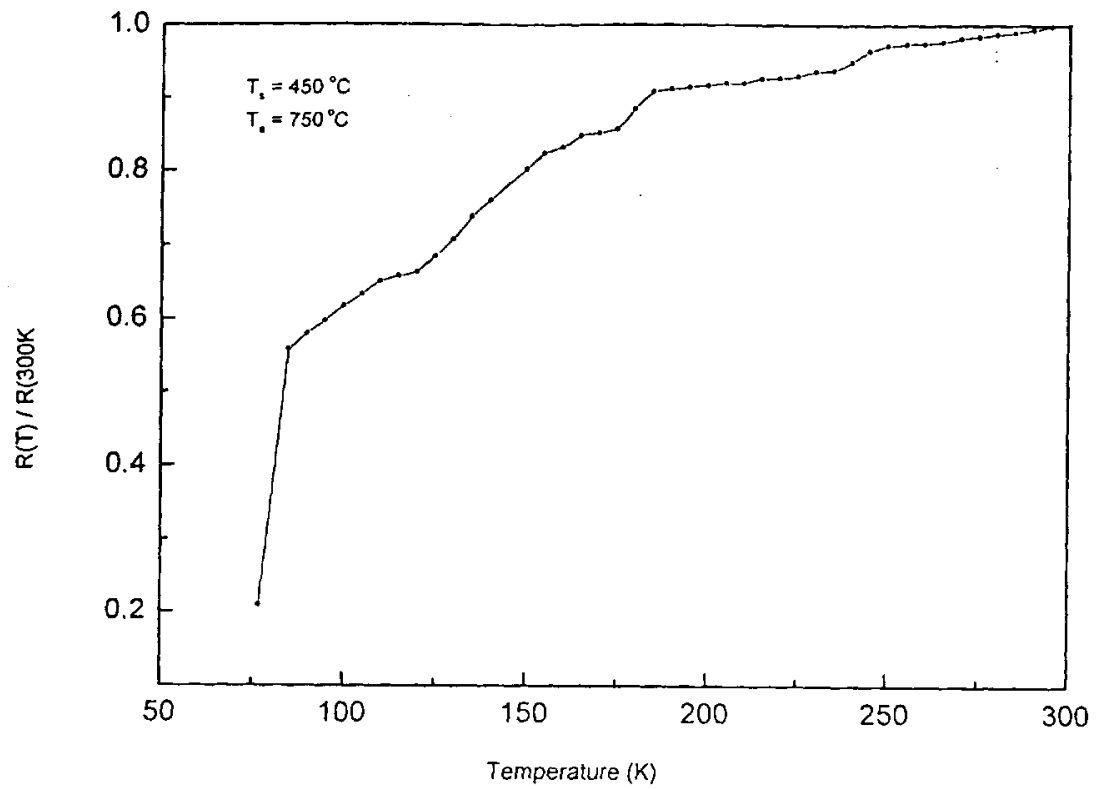

Fig. (5) : Temperature dependence of normalized resistance for YBCO films on Si single crystal substrate $\left(\mathrm{T}_{\mathrm{s}}=450{ }^{\circ} \mathrm{C}, \mathrm{T}_{\mathrm{a}}=750{ }^{\circ} \mathrm{C}\right)$. 
study, where these samples showed low crystallinity. As the annealing temperature increases to $\mathrm{T}_{\mathrm{a}} \geq 850{ }^{\circ} \mathrm{C}$, the superconducting characteristics were observed with $\mathrm{T}_{\mathrm{c}}$ in the range between 98 and $125 \mathrm{~K}$. In general, increasing the annealing temperature to $950{ }^{\circ} \mathrm{C}$ give better results, with the optimum and unexpected behaviour of sharp transition $\Delta \mathrm{T}=0.5 \mathrm{~K}$ at $\mathrm{T}_{\mathrm{c}}=125 \mathrm{~K}$ for the sample deposited at $\mathrm{T}_{\mathrm{s}}=500{ }^{\circ} \mathrm{C}$ as shown in Fig.(6). This is accompanied by the formation of crystalline phase with the proper preferred orientation (c-axis normal to the film surface) as proved by XRD analysis. Thus, it seems that these conditions result in a homogeneously strained film and its annealing leads to recrystalization with the formation of thermodynamically more stable orientation as concluded by Brecht et al. [11]. Also, the interdiffusion under the proposed preparation condition $\left(\mathrm{T}_{\mathrm{s}}=500^{\circ} \mathrm{C}\right.$ and $\left.\mathrm{T}_{\mathrm{a}}=950^{\circ} \mathrm{C}\right)$ seems to result in an optimum $\mathrm{Si}$ concentration at which the observed high $\mathrm{T}_{\mathrm{c}}$ is obtained.

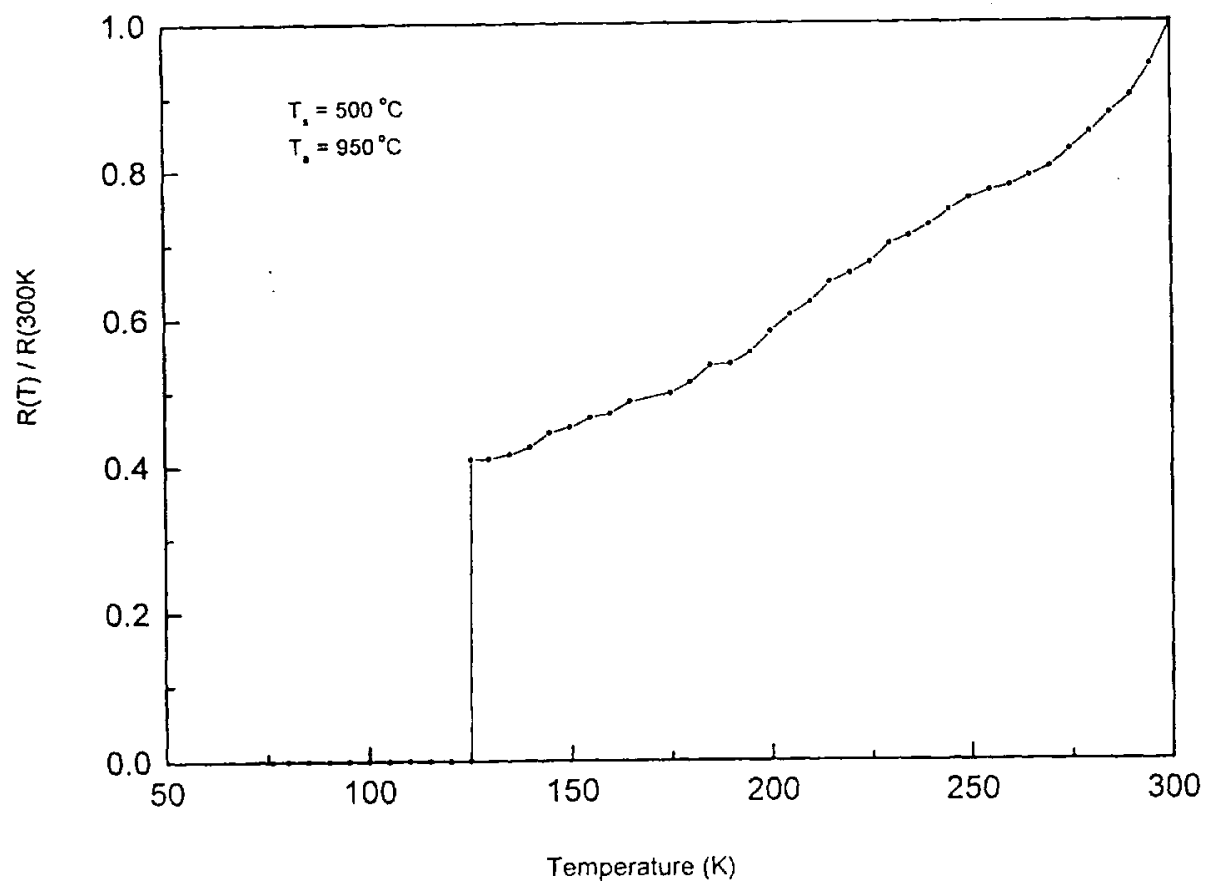

Fig. (6): Temperature dependence of normalized resistance for $\mathrm{YBCO}$ films of thickness $=85 \mathrm{~nm}$ on $\mathrm{Si}$ sigle crystal substrate $\left(\mathrm{T}_{\mathrm{s}}=500^{\circ} \mathrm{C}, \mathrm{T}_{\mathrm{a}}=950^{\circ} \mathrm{C}\right)$.

The resistance variation with temperature for a thick film $(125 \mathrm{~nm})$ of the same deposition condition as above is shown in Fig.(7). It can be seen that increasing the film thickness deteriorate the superconducting characters. On the other hand, prepared films of thickness less than $80 \mathrm{~nm}$ did not show superconducting behaviour. It is also worth to mention that the strain formation 
and release depend on the interplay of all the preparation parameters and this plays an important role in the oxygenation of the film [11], and consequently the superconducting behaviour. Therefore, the optimum thickness is about 85 $\mathrm{nm}$ for the above mentioned preparation conditions to get continuous and homogenous film with high critical transition temperature and sharp transition width.

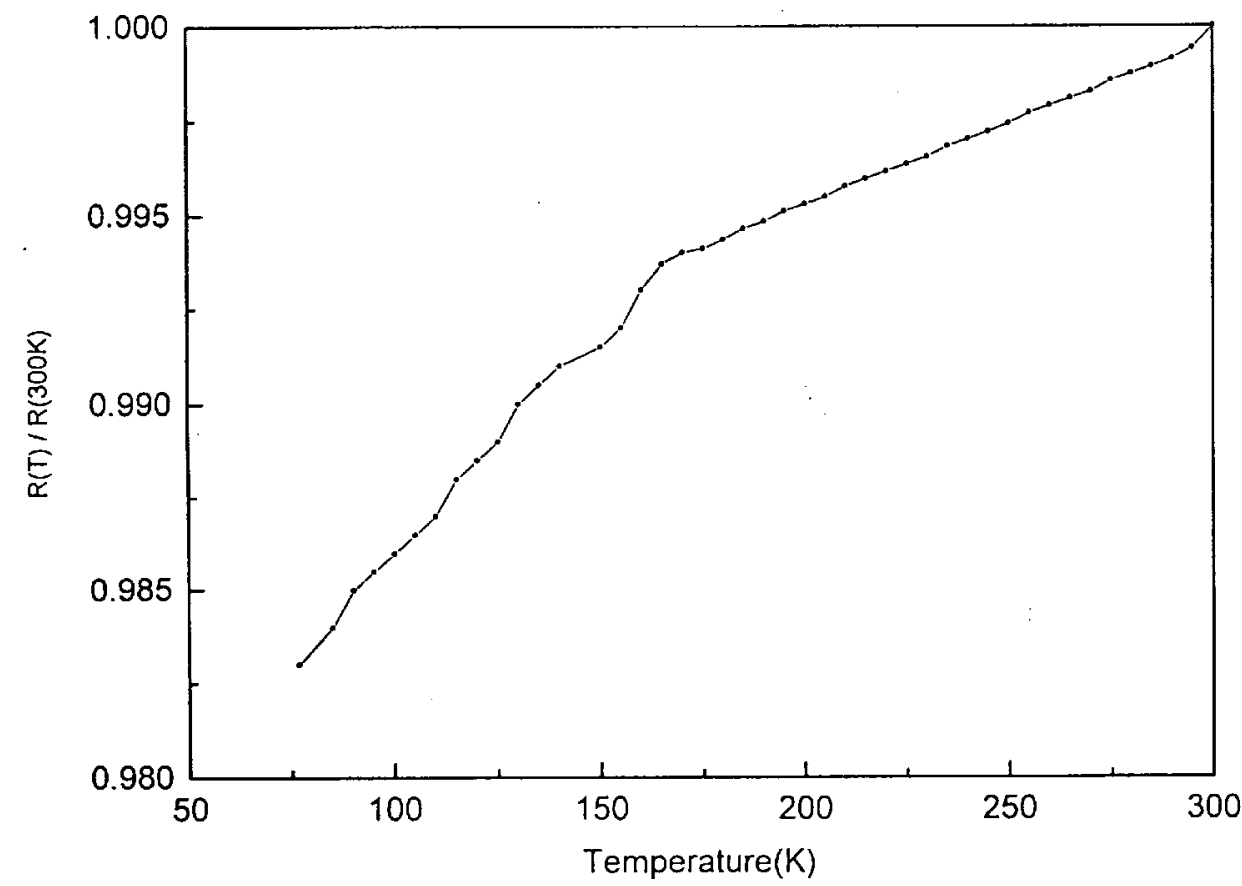

Fig. (7) : Temperature dependence of normalized resistance for YBCO films of thickness $=125 \mathrm{~nm}$ on Si substrate $\left(\mathrm{T}_{\mathrm{s}}=500^{\circ} \mathrm{C}, \mathrm{T}_{\mathrm{a}}=950^{\circ} \mathrm{C}\right)$.

\section{Conclusions}

Flash evaporation technique can be used successfully to prepare YBCO thin films with high superconducting quality (high $\mathrm{T}_{\mathrm{c}}$ up to $125 \mathrm{~K}$ and sharp transition of $\Delta \mathrm{T} \cong 0.5 \mathrm{~K}$. Also, certain concentration of Si doping $(<0.5$ at \%) may enhance the superconducting characteristics of YBCO thin films. Not only the temperatures of the substrate during deposition and post annealing are the effective parameters in superconductor thin films but also the film thickness is of vital importance. Using Si wafer substrate, the best quality can be achieved at critical thickness of $85 \mathrm{~nm}$ for films deposited at substrate temperature of $500{ }^{\circ} \mathrm{C}$ and annealed at $950^{\circ} \mathrm{C}$. 


\section{References}

1. J.G. Bednorz, K.A. Müller, Z. Phys. B64 (1986) 189.

2. Y.J. Tian, S. Linzen, F. Schmidl, A. Matthes, H. Schneidewind, P. Seidel, Thin Solid Films 338 (1999) 224.

3. R.E. Liluo, C.J. Muenchausen, J.R. Maggiore, J.R. Jimenez, L. Schowalter, Appl. Phys. Lett. 58 (1991) 419.

4. A. Kumer, J. Narayan, Appl. Phys. Lett. 59 (1991) 1785.

5. L. Mechin, J. Villegier, G.Rolland, F. Laugier, Physica C 269 (1996) 124.

6. R.A. Young, "The Rietveld Method", International Union of Crystallography Monographs on Crystallography (5), Oxford Univ. Press, Oxford, 1993, p.1.

7. R.D.L. Kristensen, S.N. Sahu, D. Haneman, Solar Eng. Mat. 17 (1988) 329.

8. J. Azoulay, Phys. Rev. B44 (1991) 7018.

9. R.A. Young, A. Sakthivel, T.S. Moss, C.O. Paiva-Santos, "Program DBWS-9411 for Rietveld Analysis of X-Ray and Neutron Diffraction Patterns", School of Physics, Georgia Inst. Tech., Atlanta, Ga, (1995).

10. Ji. Chunlin, Cui. Chuanmeng, W. Kuihan, S. Liu, G. Zeng, G. Zhang, C. Qian, W. Bain, Z. Fan, Q. Xue, Solid State Communications 65 (1988) 859.

11. E. Brecht, J. Reiner, M. Rodewald, G. Linker, Thin Solid Films 319 (1998) 202. 